\title{
BMJ Open The Only Eye Study (OnES): a qualitative study of surgeon experiences of only eye surgery and recommendations for patient safety
}

\author{
Lee Jones, ${ }^{1,2}$ Deanna J Taylor, ${ }^{1}$ Freda Sii, ${ }^{2,3}$ Imran Masood, ${ }^{2,4}$ David P Crabb, ${ }^{1}$ \\ Peter Shah ${ }^{2,3}$
}

To cite: Jones L, Taylor DJ, Sii F, et al. The Only Eye Study (OnES): a qualitative study of surgeon experiences of only eye surgery and recommendations for patient safety. BMJ Open 2019;9:e030068. doi:10.1136/ bmjopen-2019-030068

- Prepublication history and additional material for this paper are available online. To view these files, please visit the journal online (http://dx.doi. org/10.1136bmjopen-2019030068).

Received 28 February 2019 Revised 01 August 2019 Accepted 18 September 2019

Check for updates

(c) Author(s) (or their employer(s)) 2019. Re-use permitted under CC BY-NC. No commercial re-use. See rights and permissions. Published by BMJ.

${ }^{1}$ Division of Optometry and Visual Science, School of Health Sciences, City, University of London, London, UK

${ }^{2}$ Birmingham Institute for Glaucoma Research, Birmingham, UK

${ }^{3}$ Queen Elizabeth Hospital Birmingham, Birmingham, UK ${ }^{4}$ Birmingham and Midland Eye Centre, Birmingham, UK

Correspondence to

Lee Jones; lee.jones@city.ac.uk

\section{ABSTRACT}

Objective Performing surgery on patients with only one seeing-eye, where complications may result in catastrophic vision loss, presents unique challenges for the ophthalmic care team. There is currently no evidence regarding how surgeons augment their care when treating only eye patients and no guidelines for how these patients should be managed in hospital eye services. This study aimed to explore ophthalmic surgeons' experiences of only eye surgery and perceptions of current practice.

Design and participants Ten ophthalmic surgeons were asked to relate their experiences and views on performing only eye surgery in indepth, semistructured interviews. Interviews were audio-recorded and transcribed. Qualitative data were subjected to thematic analysis to identify key themes.

Setting Hospital eye service.

Results Five key themes emerged relating to surgeons' experiences and perceptions of only eye surgery: (1) differences in approach to consent, (2) strategies for risk reduction, (3) unmet training needs, (4) value of surgical mentor and (5) emotional impact of unsuccessful outcomes. Recommendations for improving the surgical journey for both the patient and the surgeon related primarily to better recognition and understanding of the complexities inherent with only eye surgery.

Conclusions Outcomes of only eye surgery may be improved through a number of methods, including development of purpose-designed training fellowships, adoption of stress-reducing strategies and enhancement of available support services. The findings identify emerging themes unique to only eye surgery and the need for guidelines on the provision of care for these highstakes surgical patients.

\section{INTRODUCTION}

All ophthalmologists will have patients under their care who have effectively only one seeing-eye. The fellow eye may have suffered severe vision loss from causes including trauma, surgical complications and advanced disease, or may have long-standing poor visual function from dense amblyopia. Patients with one seeing-eye ('only eye') are
Strengths and limitations of this study

This is the first study to qualitatively investigate surgeons' experiences of performing only eye surgery.

- Semistructured interviews provided the opportunity to gain knowledge about a wide range of aspects of only eye surgery.

- The broad range of identified themes provides basis for further evidenced-based research.

- Limitations include a relatively small number of surgeons interviewed who were all glaucoma surgeons.

always a concern for their ophthalmologists, but particularly when the better-seeing eye requires surgical intervention. This better eye may, for example, have sight-threatening glaucoma or may have an urgent problem, such as severe, uncontrolled intraocular pressure, acute macula-threatening retinal detachment, or a slowly progressive, nonurgent problem such as cataract. In moving into a surgical zone, both the patient and the surgeon are faced with the fact that surgical complications may result in sudden, total and permanent loss of vision, with life-changing consequences. Loss of vision in an only (ie, 'better') eye can have a significant impact on patients' quality of life (QoL) ${ }^{1-7}$ It is for this reason we believe that only eye surgery is appropriately considered 'high-stakes' surgery.

Incisional ocular surgery generally carries a low complication incidence rate. ${ }^{8}$ Yet sight-threatening complications such as postoperative infection and haemorrhage cannot be discounted, and unfortunately do occur. ${ }^{9} 10$ The National Ophthalmology Database reports on all National Health Servicefunded cataract surgeries in England and Wales. ${ }^{10}$ The 2018 audit highlighted intraoperative complications in $3.2 \%$ of all recorded procedures, the most prevalent being 
posterior capsular rupture (PCR). Over 3000 patients with PCR had postoperative visual acuity (VA) of 6/60 or worse, that is, unable to read the top line of a typical VA chart. Postoperative complications were more prevalent, with 1 in 20 (over 8000) eyes having at least one complication. Glaucoma randomised clinical trials report serious complications, including retinal detachment, suprachoroidal haemorrhage and endophthalmitis occurring in over one in five tube shunt and trabeculectomy patients. ${ }^{11}$ Approximately half of patients experiencing a complication lost greater than two lines on Snellen VA. In other words, if these were only eye patients with very good VA (eg, Snellen 6/6) before surgery, they certainly would no longer satisfy the criteria for safe driving eyesight and would lose their driving licence. Serious complications have been reported in only eye surgery. ${ }^{12} 13$

Researchers have recognised the unique impact of ophthalmic surgery on patients' psychological wellbeing. ${ }^{1415}$ Indeed, only eye patients are often particularly fearful, citing blindness and surgical complications as primary concerns. ${ }^{16}$ Furthermore, perceived stress among surgical staff is heightened when operating on complex or high-risk patients, ${ }^{17}$ and only eye surgery often fits both these criteria. Thus, research into how surgeons approach only eye surgery, such as strategies for risk reduction, is warranted. Surgeons can provide valuable insight into the realities of performing these high-stakes procedures, the challenges to overcome, potential strategies for effective coping and service delivery issues. The purpose of this study was to explore ophthalmic surgeons' experiences of only eye surgery, with the aim to improve the journey for both the patient and the surgeon and to identify factors that could enhance surgeon resilience.

\section{METHODS}

\section{Sampling and recruitment}

As this study adopts an inductive approach, we do not seek generalisability based on large sample sizes, but rather the appropriateness of the sample to yield a meaningful balance between thick data and rich data. ${ }^{18}$ Therefore, we conducted interviews with 10 ophthalmic surgeons (see table 1). Purposive sampling was used whereby surgeons who were known to perform only eye surgery were invited to participate. Ten surgeons were approached, all of whom agreed to participate. The majority of the surgeons worked within large general hospitals or were based in a specialist eye hospital.

There are currently no standardised definitions for what constitutes an only eye, but when considering from both patient and surgical perspectives, one could use characteristics that focus on the impact of the loss of the eye. The practical working definition used for this study was: 'An eye where significant loss of vision in this eye would be deemed life-changing with profound impact on the QoL by both the patient and surgeon'. The vision in the fellow eye (usually $<3 / 60$ or worse \pm end-stage visual field loss) was considered insufficient to maintain the patient's

\begin{tabular}{lllll}
\hline $\begin{array}{l}\text { Table } 1 \\
\text { interview date }\end{array}$ & Participant characteristics listed in order of \\
\hline ID & Sex & Specialty & Level of training & Location \\
\hline P1 & Male & G; AS & Consultant & UK \\
P2 & Male & G; AS & Consultant & Non-UK \\
P3 & Male & G; AS & Consultant & Non-UK \\
P4 & Male & G; AS & Consultant & UK \\
P5 & Male & G; AS & Consultant & Non-UK \\
P6 & Male & G; AS & Consultant & UK \\
P7 & Female & G; AS & Specialist registrar & UK \\
P8 & Female & G; AS & Consultant & UK \\
P9 & Female & G; AS & Consultant & Non-UK \\
P10 & Male & G; P & Consultant & UK \\
\hline
\end{tabular}

AS, anterior segment; G, glaucoma; P, paediatric.

current independent lifestyle and visual QoL. Loss of the only eye would likely result in a substantial impact on areas such as occupation and potentially result in a need for long-term social care, or have significant impact on family members required to help with caregiving.

\section{Data collection}

Semistructured, audio-recorded, face-to-face interviews were conducted by a male university-based $\mathrm{PhD}$ researcher (LJ) trained in collecting qualitative data. Details of the interview topic guide development are shown in figure 1. Data were collected between November 2017 and April 2018. The median (IQR) interview duration was 35 (31-40) min. Interviews were primarily carried out oneto-one in clinic rooms within the hospital eye service. The researcher corresponded with participants via email and telephone during recruitment and had met some previously through patient and public involvement events. We used interviews as this method is particularly useful when little is already known about the study phenomenon, such as in the field of only eye surgery. Interviews are also appropriate for exploring potentially sensitive topics, like surgical experience. Semistructured interviews consist of several key questions that help define areas to be explored, but also allow the interviewee to diverge in order to pursue an idea or response in more detail. This interview format is frequently used in healthcare-related research, as it provides participants with some guidance on what to talk about, which many find helpful. The flexibility of this approach, particularly compared with structured interviews or surveys, allows for the discovery or elaboration of information that is important to participants, but may not have previously been thought of as pertinent by the research team.

\section{Patient and public involvement}

The Only Eye Study (OnES) is a series of research projects to investigate only eye surgery. This is the first research output in the series and reports surgeon experiences of 


\section{Advisory group* meet to identify potential aspects of surgery to be discussed}

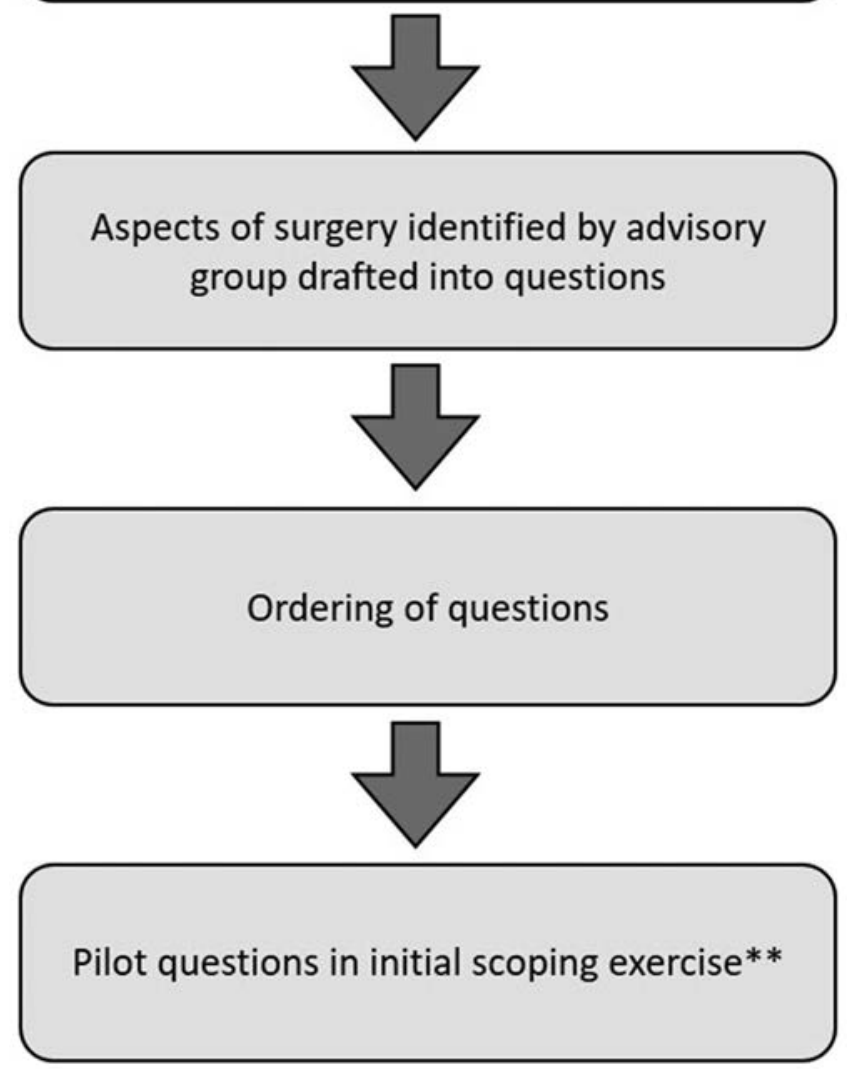

Figure 1 Process of interview topic guide formation. ${ }^{*}$ Advisory group consisted of only eye patients, consultant ophthalmologists, ophthalmologists-in-training, a psychologist and an ophthalmic research nurse, and established researchers in the field of ophthalmology. ${ }^{* \star}$ Scoping exercise consisted of a preliminary pilot interview where suitability of interview questions was assessed.

only eye surgery. The outcomes of the patient interviews will be described in a subsequent report. As shown in figure 1, patients with experience of only eye surgery were included in the advisory group who helped to develop the interview topic guide for this study. Following data analysis, a 'Bridging the Gap' event was hosted at Queen Elizabeth Hospital Birmingham which gave the opportunity to disseminate the research findings among surgeons, patients and their carers.

\section{Data management and analysis}

The study was designed and reported following the guidance of the Consolidated criteria for Reporting Qualitative research (COREQ). ${ }^{19}$ Interviews were coded with manual and computer-based methods (NVivo V.11; QSR International, Cambridge, Massachusetts, USA) using thematic analysis. ${ }^{20}$ Open coding was used when analysing the data where patterns in participants' responses were recorded. These patterns were further explored by grouping responses into similar categories both within and across interviews, and finally were grouped into common themes which best described the content of the data. The study was designed to recruit 10 participants, and so no direct decision was taken to cease data collection; however, similar themes continued to emerge in the latter interviews, and so it is likely that 'data saturation' was achieved. Two members of the research team (LJ and DJT) read and reread the transcripts and independently developed preliminary codes based on impressions of recurring themes. Inter-rater reliability was assessed using the kappa coefficient $(\kappa)$ and was acceptable between the two coders $(\kappa=0.46)$. There is debate in the literature regarding the sufficiency of the kappa statistic; however, scores between 0.40 and 0.75 typically reflect fair to good agreement beyond chance. ${ }^{21}$ Following individual interpretation, the authors met to reflect on the entire interview data and discuss differences of opinion regarding themes. Once in agreement of the meaning of quotes and suitability of coding choices, a coding framework was created where the key themes were finalised.

A number of methods were employed to ensure the study had appropriate rigour and maintained research integrity. As shown in figure 1, the project was steered by a number of relevant stakeholders including both patients and surgeons who assisted in the study design and analysis. We piloted the interview topic guide with two surgeons, leading to a slightly revised final topic guide. All members of the research team, including surgeons, were involved in establishing the appropriateness of the generated codes. We contributed to improving descriptive validity through the use of a strict verbatim transcription service. In addition, member-checking was used with three interviewees to assess our choice of coded themes in an attempt to improve the reliability and trustworthiness of our findings. Finally, we ensured the design, conduct and reporting of the study followed the COREQ guidelines. ${ }^{19}$

\section{RESULTS}

Five key themes emerged relating to 'Differences in approach to consent', 'Strategies for risk reduction', 'Unmet training needs', 'Value of surgical mentor' and 'Emotional impact of unsuccessful outcomes'. Quotes taken from the transcripts are shown in the following sections and illustrate key themes that came from the interviews. All excerpts are annotated with a code given to the corresponding surgeon. Additional quotes are provided in online supplementary appendix 1.

\section{Differences in approach to consent}

Participants described concerns regarding consent and discussion of material risk in only eye surgery. While participants agreed that consent prior to only eye surgery is essential, there were differences regarding how extensive the information provided should be. In some cases, participants expressed the importance of sufficiently 
disclosing the risks involved with treatment, and that consent should be focused on what is material to the patient.

The patient needs to know and understand what it means to be totally blind. If the patient does go blind and they're not fully aware of what it means to be totally blind, they'll be extremely distraught. (P4)

There are risks with this surgery, and you talk about the risks, including that the operation itself might tip them over the edge and make them lose their vision. (P6)

Yet some participants described aversion to placing too much emphasis on potential for total vision loss in only eye surgery, suggesting that it can be counterproductive to focus on possible negative outcomes of surgery.

You don't want to really hammer home the point that this is their only eye because they're already anxious, and most people are aware if they had a complication in their only eye the stakes are much higher. So I don't think it's useful or necessary to dwell on it too much. (P7)

Moreover, participants explained that some patients appear to prefer not to know about risks of surgery.

There are patients who don't want to take part in that decision; they leave it entirely into your own hands. That's fair; that's good enough. (P5)

Participants explained how they attempt to demonstrate risks of surgery to patients. Practical techniques used to exemplify what life might be like for the patient if the surgery was unsuccessful were described.

We patch them up for three hours in clinic and we sit them outside so they are totally blind. So they've been totally blind for three hours when we consent them. (P1)

The idea of a dual consent process was described, whereby a second or third opinion is sought before agreement to proceed. It was suggested that dual consent can help patient decision to proceed with surgery, and reassure the surgeon if their colleagues agree the intervention is needed.

We're increasingly doing dual consenting. Patients that are high-risk only eye, it's always useful if you've got two people doing consent. Certainly, joint clinics and multi-disciplinary clinics allow for the opportunity. (P4)

\section{Strategies for risk reduction}

Participants gave details about strategies they incorporate in only eye surgery to optimise outcomes. Several preoperative strategies were highlighted, typically regarding logistics. One strategy was to ensure availability of correct and optimal surgical instruments.
If you don't ask for the best instruments you will get given an average set, which usually has one or two things broken. There'd be a limited number of perfect instrument sets within the theatre, so I have to make sure that I've got those. I have a special only eye tray only I'm allowed to use. It's called the only eye tray. (P1)

Another method was for the surgeon to adopt physical and mental relaxation techniques, such as task visualisation, whereby the surgical procedure is visualised and mentally performed prior to surgery.

I will visualise the steps that I will go through. Visualise what may go wrong, and what I will do to undo that. I visualise even the routine, the basic steps. (P8)

A positive and optimistic attitude before operating was also considered a benefit in only eye procedures.

If you think, I'm going to do that case successfully and it'll be great, I think you'd feel a lot better than if you think: oh imagine if I have a complication. (P7)

In other instances, surgeons relied on faith in order to cope with the stresses of surgery.

Before I operate, I pray. I take on very difficult and crazy cases where sometimes I'll be doing this surgery for the first time in my life. I do it because I have faith, and I pray, and I believe God helps me. (P4)

One suggestion for reducing risk intraoperatively was to ensure only eye patients were operated on by teams of highly experienced surgeons, rather than a single surgeon.

There's been instances where another surgeon being there has made a crucial difference to the outcome, because they've spotted things that I may not have spotted because I was busy with something else. (P4)

Yet the consensus was that only eye patients are often operated on by just one surgeon.

It's a good idea to do difficult cases together, but because our outcomes are usually not lethal, it's not about life or death, we can't finance a second surgeon. (P3)

In many branches of surgery, high-risk cases where the impact of failure is massive, are done by two surgeons, or teams of surgeons. In ophthalmology often there's just one surgeon. I wonder if we are missing something just because it's a small organ. (P1)

Participants also expressed preference for avoiding this approach and warned of potential hazards of high-stakes procedures being performed by teams.

I wouldn't prefer having someone around in the same operating theatre for moral support. On the contrary, I think I'd be distracted. (P5) 
Finally, participants discussed postoperative strategies for risk reduction. Frequent and timely follow-up appointments were often scheduled for only eye patients. In addition, the issue of failure to rescue an only eye from surgical complications was addressed, with suggestion for a protocol to reduce postoperative threats to only eye patients.

You do more when you know it's an only eye. That's not to say you care less [in non-only eye surgery]. But knowing it's an only eye, you just add extra steps. In the follow-up, you see them sooner, more frequently. (P8)

A lot of times, things go wrong because of a failure to rescue a simple problem. If an only eye patient has problems following surgery, they need to be seen by a senior doctor, and that should be part of the protocol in a hospital. (P4)

\section{Unmet training needs}

Many participants correlated lack of surgical experience with concerns about care provision for only eye patients. For example, senior surgeons expressed doubt that the current training programme in ophthalmology provides sufficient exposure to high-stakes patients. One explanation for this dearth in experience was increased time restrictions on surgical activity.

The trainees are not getting the training. The number of cases are dropping, they are shortening the number of training years, so trainees are being compressed in both ways. (P8)

Time restrictions were perceived to have ramifications for future aptitude and resilience of ophthalmologists-in-training.

Looking at the last five years, I can't think of a single trainee who I feel had the necessary technical ability, bravery, and surgical resilience to be safely allowed to do these cases. I wonder what's going to happen when they become young consultants. I don't think they will have the necessary skill set. (P1)

They will become a consultant with probably less than $50 \%$ experience as the previous generation, so that will be a problem. (P8)

There was a consensus that hospital eye services delegate only eye patients to experienced staff only.

The head of the department or the Medical Director would do all the only eyes themselves, just to take responsibility for it. (P3)

You wouldn't want a trainee doing an operation on an only eye, that would be inappropriate and I think we'd be doing the wrong thing by the patient if that was the case. (P10)

Participants believed more needs to be done to prepare ophthalmologists-in-training for only eye procedures. There were recommendations on overcoming this issue, such as specialised training programmes for only eye care.
It's important we identify the best people and they are given focused training, focused mentoring by senior surgeons who do that kind of surgery, and gradually get them to that level. In this kind of surgery, you've got to get the best people, because patients only have one chance. $(\mathrm{P} 4)$

\section{Value of surgical mentor}

Participants emphasised that becoming a successful only eye surgeon relies heavily on good mentorship. On several occasions, participants stressed the value of having a mentor for transferring knowledge to assist in personal, professional and educational development.

You need to be a good surgeon to do only eyes with confidence, and that means you need to have good teachers, who teach more than just the technique. A good mentor, I would say is key. (P3)

Everyone needs to have a good mentor who can advise them, I think that's probably the most important thing. My advice to anyone who does only eye surgery is to find a mentor who's been doing this kind of surgery, talk to them, learn from them, how they approached it. (P4)

Yet a barrier to effective mentoring was time commitment issues, where there was advocacy for formal recognition of mentoring programmes to alleviate these constraints.

If trainees have a mentor who has been through lots of things, they can come through for support when things don't go right. But obviously that role has to be recognised. (P8)

\section{Emotional impact of unsuccessful outcomes}

Among our participants, some had experienced 'losing' an only eye, resulting in catastrophic loss of vision for the patient. In these cases, participants described being burdened with a sense of personal responsibility and expressed how they have shouldered the blame.

We lost a true only eye, he went blind. It doesn't leave you, I still feel like I could have done something different. I feel like if the time was slightly different, if we weren't so stressed, if we weren't so under pressure, I think we would have said there's something not right. I still feel partly responsible for him. (P8)

Participants expressed concern over how losing an only eye would affect their career and the psychological sequelae of such an event.

I'm lucky not to have had an only eye disaster, yet. I'll probably remember that for the rest of my life. (P10)

Participants described how they have witnessed changes in colleagues' demeanour after adverse events following only eye surgery.

Colleagues who've gone through that experience, you can see it in their face and their body language 
how damaging it can be. At your hands a life-changing event for the worse has occurred. That immediacy, actually, is one of the unique burdens of being a surgeon. (P10)

For some of them, it will haunt them and they might not ever perform at their peak again. (P8)

There was recognition of the need for systems to support surgeons after losing an only eye.

There needs to be a better support mechanism. If someone has lost an only eye and they're distraught by it, they need a mentor to talk to, someone who has lost an only eye. (P4)

Yet many perceived a lack of pathways to find professional support services in the event of losing an only eye. Participants also noted barriers to seeking out such services.

There's no guidance on how surgeons can seek help for themselves when incidents like this happen. (P8)

We're very busy. We don't have time to do that [seek support services]. Something else would have to give. (P6)

The thing is, we're too busy. That's taking time up and it's not strictly urgent. It can be pushed to the bottom of the pile, even though I don't think that's the right thing to do. $(\mathrm{P} 7)$

\section{DISCUSSION}

Surgery on an only eye can be described as high-stakes; if unsuccessful, patients may become blind for the rest of their lives. As such, only eye surgery can be challenging for surgeons and the ophthalmic care team. We sought to explore ophthalmic surgeons' experiences of performing high-stakes procedures on patients with only one seeing-eye.

Our findings highlight differences in how surgeons disclose material risks in only eye surgery. Participants stressed the importance of patients' understanding of the risks of surgery, regardless of how unlikely adverse outcomes may be. Yet other participants voiced concerns over a heavy focus on risks of vision loss, as surgery is generally successful. This discordance is pertinent given the landmark change in the position of the Supreme Court regarding informed consent. ${ }^{22}$ Until recently, the UK Supreme Court followed the principles of the Bolam Test. Such principles state that, in the event of surgical complications, a surgeon would not be deemed negligent if they had acted the same way other competent surgeons would have. ${ }^{23}$ However, this paternalistic approach to medicine is no longer tolerated, as demonstrated by the introduction of the Modified Montgomery Test. ${ }^{24}$ This standard of care obliges surgeons to provide sufficient information to patients, including disclosure of risks of proposed treatment. In medicine, there is concern over the use of a 'one-size-fits-all' approach applied to heterogeneous populations. ${ }^{25}$ For example, greater material risk should be attached to surgery on an only eye, as opposed to the same surgery on a patient with good bilateral vision. Yet participants expressed aversion to appearing pessimistic when discussing surgical risks, a belief in contrast to the principles of the Modified Montgomery Test. Methods of demonstrating risks of only eye surgery included occlusion of the only eye. Our results indicate variances between surgeons regarding discussion of material risks in only eye surgery, suggesting the principles of the Modified Montgomery Test are yet to be fully recognised in this area of ophthalmology.

Our study has notable findings regarding minimising risk in only eye surgery. One risk reduction strategy was to ensure all surgical equipment was of highest possible quality. Quality control exercises highlight a strikingly high percentage of defective surgical instruments delivered to UK hospitals, ${ }^{26}$ and operating room incidents with potential to affect quality of care are most commonly equipmentrelated. ${ }^{27}$ Indeed, surgical environments are often busy and surgeons are rarely afforded time to optimise all possible variables prior to surgery, and even meticulous scrutiny will not eliminate risk. Yet optimal instruments were deemed an important preoperative strategy to minimise risk in this study. There is advocacy for patients requiring specialised care, such as only eye patients, to be managed under larger, high-volume hospitals, where they are more likely to be treated using the most cutting-edge equipment. ${ }^{28}$ However, consideration must be given to what is the most suitable arrangement for the patient. Our results raise the question as to whether specialised resources are required for treatment of only eye patients.

It was acknowledged that only eye procedures can be particularly stressful events for surgeons. Self-reported anxiety is typically higher when the procedure is highstakes. ${ }^{17}$ Stress-reducing strategies, such as mental imagery, optimistic attitude and spiritual activities, were described as a means of reducing performance anxieties and bolstering a relaxed mental state before surgery. Psychological relaxation strategies are reported to enhance surgical performance. ${ }^{29}$ Moreover, evidence indicates that surgeons who undertake mental skills training have better outcomes on measures of anxiety. ${ }^{30}$ Our results identify coping strategies used by surgeons before only eye surgery to optimise performance during stressful situations.

Our findings introduced the concept of only eye surgery being performed by two or more surgeons. This intraoperative strategy for risk reduction was described as an opportunity for another expert to critique the procedure, in an attempt to ensure nothing is missed. However, some participants perceived this approach to be counterproductive, suggesting team procedures can lead to adoption of more risk-averse or overly foolhardy behaviours. The advocacy for only eye procedures performed by two or more surgeons echoes how exceptional cases are managed in other fields of medicine, such as cardiothoracic surgery. In this specialty, implementation of a Star Chamber, whereby surgeons refer complex or high-stakes patients to the Star Chamber which assesses what the patient should be offered, has been used 
in an attempt to improve surgical outcomes. ${ }^{31}$ If the Star Chamber recommends surgery, it is a requirement that the procedure is performed by a minimum of two consultants. Other disciplines in the UK are considering implementation of a Star Chamber ${ }^{32}$; however, there appears to be no such movement in ophthalmology. Yet such initiatives as the Star Chamber may help to minimise intraoperative risks during only eye surgery.

Participants reported following only eye patients up closely postoperatively, scheduling more frequent appointments to ensure that complications are quickly addressed. In only eye surgery, an appropriate and timely response is essential to prevent failure to rescue. Although many participants reported closer follow-up of only eye patients, evidence suggests that patients with monocular vision undergoing surgery on their only eye do not receive more telephone calls or clinic visits preoperatively or postoperatively. ${ }^{33}$ Analysis of doctor-patient interaction, such as time spent in clinic, may provide useful information regarding differences in follow-up patterns between only eye and nononly eye patients; this would be an interesting avenue for future research.

Participants described how medical training in the UK has experienced dramatic reform and expressed concerns over how this may affect standards of care in ophthalmology. Changes in educational theory ${ }^{34}$ and the European Working Time Directive ${ }^{35}$ have limited training opportunities for ophthalmologists-in-training. As a result, procedures such as trabeculectomy feature less often in trainees' timetabled clinical activity. ${ }^{36}$ Work-hour restrictions and a demise of the 'mentor' model in medical training may have damaging consequences for acquisition of technical skills and surgical resilience. ${ }^{37}$ Indeed, consultant surgeons have reported concerns over the capabilities of the newer generation of trainees and how this may impact patient care. ${ }^{38}$ Although progress in technology has led to the advent of valuable training opportunities, such as 'wet-lab' simulations, ${ }^{39}$ such environments are unable to mimic the true reality of operating on a patient's only eye. Furthermore, recent evidence suggests many teaching programmes have not implemented specific policies for ophthalmologists-intraining performing cataract surgery on only eye patients. ${ }^{40}$ Our participants stressed the essentiality of combating these training barriers and gave suggestions for purposedesigned training programmes for complex procedures. Such programmes may enable appropriate access to high-stakes patients and nurture the learning processes for ophthalmologists-in-training. This finding spotlights concerns with surgical training in ophthalmology, a problem first identified almost two decades ago. ${ }^{41}$ If this trend continues, there may be necessity for specific training fellowships to gain clinical competency, and we propose that only eye training must not be overlooked.

Another emerging theme was the importance of mentoring in only eye surgery. Participants described how a good mentor has helped them to become an effective only eye surgeon. Typically, a mentor will be a senior member in the field who guides a trainee professionally and personally by facilitating learning through observation and modelling. ${ }^{37}$ There is concern that mentoring has become a lost art in medicine, ${ }^{42}$ and participants in our study explained that a mentor can offer significant support when caring for only eye patients and formal recognition of mentoring may be needed. In line with previous research, our results highlight barriers to mentorship as a lack of formal recognition of the role, resulting in time commitment issues and a scarcity of appropriate mentors. ${ }^{43}$ Fostering of strong relationships between the mentor and the trainee could play a crucial role in alleviating concerns raised in this study about training in ophthalmology and only eye surgery.

A number of participants had experienced losing an only eye, resulting in total extinction of vision for the patient. Participants described their responses to these incidents and how the psychological sequelae have impacted their career. A recurring sense of personal responsibility and blame was reported, and participants remarked on lack of formal support for surgeons when unpredicted outcomes occur. In medicine, the term 'second victim' was coined to recognise that the surgical team may suffer in the event of negative outcomes. ${ }^{44}$ Often, long-lasting emotional distress of such outcomes will affect all members of the patient's healthcare team, including surgeons, nurses and allied healthcare professionals. ${ }^{45}$ Proposals have outlined the needs of the second victim, which include entitlement to psychological support services ${ }^{46}$ although our participants perceived a lack of avenues to seek professional support after losing an only eye. Lack of time is a primary barrier to uptake of support services. ${ }^{47}$ However, growing attention is being placed on the mental well-being of surgeons, ${ }^{48}$ and the importance of such support services as the Practitioner Health Programme is being realised. ${ }^{49}$ Still, a large number of UK hospitals remain without a policy for staff mental health support. ${ }^{50}$ Participants in our study perceived a lack of options for support in the event of poor outcomes in only eye surgery, reflecting lack of recognition and understanding of the second victim phenomenon in ophthalmology.

Many fields of medicine have adopted multidisciplinary teams to manage complex conditions. Our study suggests that only eye surgery might benefit from being performed by teams of surgeons experienced in ophthalmic surgery, as well as in the psychological preparation of patients and surgeons. Furthermore, these teams should have the resources to identify, develop and mentor inexperienced surgeons in order to succession plan and ensure skill levels are maintained. National guidelines for teams performing only eye surgery should be drafted to ensure that the risk of total blindness is reduced as far as possible.

This study is the first of its kind in ophthalmic surgery. By adopting a qualitative approach, important themes have emerged which provide an excellent basis for further work. Our participants worked in a variety of geographical locations; thus, a limitation is that 
differing work cultures may restrict the comparability between experiences. However, this can be considered a strength as we were able to capture the wide range of surgeons' experiences, including strategies which may be transferrable between countries and institutions. The study is limited in that a small number of surgeons were interviewed and they were all experienced glaucoma surgeons, primarily based in large hospital care centres, which may restrict the transferability of our findings. This may be due to the nature of glaucoma in that there may be a greater proportion of patients who are only eyed, particularly in complex glaucoma practices. It is important that future studies consider the views of less experienced surgeons.

\section{CONCLUSIONS}

The implications of losing an only eye are massive for both the patient and the surgeon. This study clearly identifies important themes that are of great relevance to surgeons who perform only eye surgery. These include risk management, training, psychology and mentoring. Further evidence-based studies are needed in each of these areas to clearly define best practice and inform guidelines to enable a safe and seamless patient journey.

\section{Twitter Lee Jones @crabblab}

Acknowledgements The authors wish to thank the advisory group who helped develop the interview topic guide, and the surgeons who provided their valuable time and thoughts in the study interviews.

Contributors LJ performed the study design, data collection, data analysis and manuscript preparation. DJT performed the data analysis and manuscript critique. FS performed the data analysis and manuscript critique. IM performed the data analysis and manuscript critique. DPC performed the study design and manuscript critique. PS performed the study design, data analysis and manuscript critique.

Funding The authors have not declared a specific grant for this research from any funding agency in the public, commercial or not-for-profit sectors.

Competing interests None declared.

Patient consent for publication Not required.

Ethics approval The study was approved by the London - Chelsea Research Ethics Committee (ref: 17/L0/1664) and conformed to the tenants of the Declaration of Helsinki. Consent from all participants was obtained prior to interview.

Provenance and peer review Not commissioned; externally peer reviewed. Data availability statement No data are available.

Open access This is an open access article distributed in accordance with the Creative Commons Attribution Non Commercial (CC BY-NC 4.0) license, which permits others to distribute, remix, adapt, build upon this work non-commercially, and license their derivative works on different terms, provided the original work is properly cited, appropriate credit is given, any changes made indicated, and the use is non-commercial. See: http://creativecommons.org/licenses/by-nc/4.0/.

\section{REFERENCES}

1 Jones L, Bryan SR, Crabb DP. Gradually then suddenly? decline in Vision-Related quality of life as glaucoma worsens. J Ophthalmol 2017;2017:1-7.

2 Peters D, Heijl A, Brenner L, et al. Visual impairment and visionrelated quality of life in the Early Manifest Glaucoma Trial after 20 years of follow-up. Acta Ophthalmol 2015;93:745-52.
3 Glen FC, Crabb DP. Living with glaucoma: a qualitative study of functional implications and patients' coping behaviours. BMC Ophthalmol 2015;15:128.

4 Kotecha A, Richardson G, Chopra R, et al. Balance control in glaucoma. Invest Ophthalmol Vis Sci 2012;53:7795-801.

5 Ramulu PY, Hochberg C, Maul EA, et al. Glaucomatous visual field loss associated with less travel from home. Optom Vis Sci 2014;91:187-93.

6 Dong LM, Childs AL, et al, Submacular Surgery Trials Research Group. Health- and vision-related quality of life among patients with choroidal neovascularization secondary to age-related macular degeneration at enrollment in randomized trials of submacular surgery: sst report No. 4. Am J Ophthalmol 2004;138:91-108.

7 Marback RF, Maia Júnior OdeO, Morais FB, et al. Quality of life in patients with age-related macular degeneration with monocular and binocular legal blindness. Clinics 2007;62:573-8.

8 Kirwan JF, Lockwood AJ, Shah P, et al. Trabeculectomy in the 21st century: a multicenter analysis. Ophthalmology 2013;120:2532-9.

9 Edmunds B, Thompson JR, Salmon JF, et al. The National survey of trabeculectomy. III. early and late complications. Eye 2002;16:297-303.

10 The Royal College of Ophthalmologists. National ophthalmology database audit key findings summary, 2018. Available: https:// www.nodaudit.org.uk/u/docs/20/iitpmzzqxi/NOD\%20Audit\% 20Summary\%202018.pdf [Accessed 27 Jun 2019].

11 Gedde SJ, Herndon LW, Brandt JD, et al. Postoperative complications in the tube versus trabeculectomy (TVT) study during five years of follow-up. Am J Ophthalmol 2012;153:804-14.

12 Rodríguez AA, Olson MD, Miller KM. Bilateral blindness in a monocular patient after cataract surgery. J Cataract Refract Surg 2005;31:438-40.

13 Eradurman C, Mehmet M, Mustafa C, et al. Results of cataract surgery in monocular patients. Glokom-Katarakt 2006;1:271-4.

14 Cross V, Shah P, Glynn M, et al. ReGAE 5: can we improve the surgical journey for African-Caribbean patients undergoing glaucoma filtration surgery? some preliminary findings. Clin Ophthalmol 2009;3:1-12.

15 Janz N, Wren PA. The Collaborative initial glaucoma treatment study interim quality of life findings after initial medical or surgical treatment of glaucoma. Ophthalmology 2001;108:1954-65.

16 Marback RF, Espíndola RFde, Santhiago MRde, et al. Cataract surgery: emotional reactions of patients with monocular versus binocular vision. Rev Bras Oftalmol 2012;71:385-9.

17 Anton NE, Montero PN, Howley LD, et al. What stress coping strategies are surgeons relying upon during surgery? Am J Surg 2015;210:846-51.

18 Tracy SJ. Qualitative quality: eight "big-tent" criteria for excellent qualitative research. Qualitative Inquiry 2010;16:837-51.

19 Tong A, Sainsbury P, Craig J. Consolidated criteria for reporting qualitative research (COREQ): a 32-item checklist for interviews and focus groups. Int J Qual Health 2007;19:349-57.

20 Braun V, Clarke V. Using thematic analysis in psychology. Qual Res Psychol 2006;3:77-101.

21 Landis JR, Koch GG. The measurement of observer agreement for categorical data. Biometrics 1977;33:159-74.

22 Edozien LC. Uk law on consent finally embraces the prudent patient standard. BMJ 2015;350:h2877.

23 The Royal College of Ophthalmologists. Consent: the reasonable patient, 20160nline. Available: https://www.rcophth.ac.uk/wpcontent/uploads/2016/02/CN-Focus-Winter-16.pdf [Accessed 10 Dec 2018]

24 Sokol DK. Update on the UK law on consent. BMJ 2015;350:h1481.

25 National Confidential Enquiry into Patient Outcome and Death (NCEPOD). Elective \& Emergency Surgery in the Elderly: An Age Old Problem, 2010. Available: http://www.ncepod.org.uk/2010report3/ downloads/EESE_fullReport.pdf [Accessed 4 Jul 2018].

26 Brophy T, Srodon PD, Briggs C, et al. Quality of surgical instruments. Annals 2006;88:390-3.

27 Wubben I, van Manen JG, van den Akker BJ, et al. Equipmentrelated incidents in the operating room: an analysis of occurrence, underlying causes and consequences for the clinical process. BMJ Qual Saf 2010;19:e64.

28 Darzi A. Healthcare for London: a framework for action. London: NHS London, 2007. http://www.nhshistory.net/darzilondon.pdf

29 Anton NE, Bean EA, Hammonds SC, et al. Application of mental skills training in surgery: a review of its effectiveness and proposed next steps. J Laparoendosc Adv Surg Tech A 2017;27:459-69.

30 Stefanidis D, Anton NE, Howley LD, et al. Effectiveness of a comprehensive mental skills curriculum in enhancing surgical performance: results of a randomized controlled trial. Am J Surg 2017;213:318-24. 
31 Nashef SAM, Surgeons NSA. Surgeons, high risk interventions and the birth of the StAR chamber. J Thorac Dis 2017;9:S426-7.

32 The Association of Coloproctology of Great Britain and Ireland. Clinical outcomes publication update for ACPGBI membership, 2016. Available: https://www.acpgbi.org.uk/content/uploads/2016/ 07/Clinical-Outcome-Publication-Update-for-ACPGBI-MembershipJuly-2016.pdf [Accessed 8 Nov 2018].

33 Rodriguez AA, Olson MD, Miller KM. Cataract surgery takes longer in functionally monocular patients. OSLI 2007;38:23-6.

34 Reznick RK, MacRae H. Teaching surgical skills--changes in the wind. N Engl J Med 2006;355:2664-9.

35 Department of Health. The European working time directive for trainee doctors -Implementation update. London: Department of Health, 2009.

36 Rodrigues IAS, Symes RJ, Turner S, et al. Ophthalmic surgical training following modernising medical careers: regional variation in experience across the UK. BMJ Open 2013;3:e002578.

37 Cope A, Bezemer J, Mavroveli S, et al. What attitudes and values are incorporated into self as part of professional identity construction when becoming a surgeon? Acad Med 2017;92:544-9.

38 Rashid A, Al-Hadithy N, Rossouw D, et al. Consultant Attitudes to 'St' Higher Surgical Trainees. Ann R Coll Surg Engl 2012;94:1-334.

39 Lee GA, Chiang MY-M, Shah P. Pig eye trabeculectomy-a wet-lab teaching model. Eye 2006;20:32-7.

40 Chen X, Zafar S, Sikder S, et al. National survey and outcomes of resident-performed cataract surgery in monocular patients in the United States. J Cataract Refract Surg 2019;45:939-45.
41 Gibson A, Boulton M, Watson M, et al. Surgical training in ophthalmology. The Lancet 2002;360.

42 Rohrich RJ. Mentors in medicine. Plast Reconstr Surg 2003;112:1087-8.

43 Entezami P, Franzblau LE, Chung KC. Mentorship in surgical training: a systematic review. HAND 2012;7:30-6.

44 AW W. Medical error: the second victim. The doctor who makes the mistake needs help too. BMJ 2000;320:726-7.

45 Ullström S, Andreen Sachs M, Hansson J, et al. Suffering in silence: a qualitative study of second victims of adverse events. BMJ Qual Saf 2014;23:325-31.

46 Denham C. Trust: the 5 rights of the second victim. J Patient Saf 2007;3:107-19.

$47 \mathrm{Hu}$ Y, Fix ML, Hevelone ND, et al. Physicians' needs in coping with emotional stressors the case for peer support. Arch Surg 2012;147:212-7.

48 Gerada C. Doctors and mental health. Occup Med 2017;67:660-1.

49 Brooks SK, Chalder T, Gerada C. Doctors vulnerable to psychological distress and addictions: treatment from the practitioner health programme. J Ment Health 2011;20:157-64.

50 Sloan D, Jones S, Evans E, et al. Implementing NICE public health guidance for the workplace: a national organisational audit of NHS trusts in England, round 2. London: Royal College of Physicians, 2014. 